\title{
Diagnosis and Treatment of Depression Following Traumatic Brain Injury
}

\author{
Theodore Tsaousides, ${ }^{1}$ Teresa A. Ashman, ${ }^{2}$ and Wayne A. Gordon ${ }^{1}$ \\ ${ }^{1}$ Icahn School of Medicine at Mount Sinai, New York, NY, USA \\ ${ }^{2}$ New York University Langone Medical Center, New York, NY, USA
}

\begin{abstract}
Depression is one of the most common psychiatric diagnoses among individuals with traumatic brain injury (TBI). Prevalence of post-TBI depression (PTBID) ranges from 12 to $60 \%$ and is generally higher than rates reported in the general population. The wide range in reported rates is attributed to methodological variability across studies, including measurement and sampling differences. Several systematic reviews have been published in the past 5 years, reporting on outcomes for depression across different classes of interventions, including pharmacological, biomedical and behavioural. The consensus across reviews is that more research is necessary to develop evidence-based practice guidelines. The present narrative review synthesises the findings of previous studies, focusing on the nature of the interventions, the eligibility criteria for inclusion and the assessment of outcome. Pharmacological studies are generally more rigorous methodologically, but provide mixed findings. Other biomedical interventions are only at the initial stages of research development, including case and pilot studies. The results of behavioural studies are positive regarding improvements in mood. However, the number of efficacy studies of behavioural interventions for depression is extremely limited. Recommendations for designing interventions are provided.
\end{abstract}

Keywords: depression, traumatic brain injury, interventions, pharmacological, psychotherapeutic, neurorehabilitation

Depression is one of the most common psychiatric diagnoses among individuals with traumatic brain injury (TBI). The prevalence of post-TBI depression (PTBID) has been documented in numerous studies, and despite significant variations in the estimates of PTBID rates, consensus exists among researchers and clinicians that PTBID remains a significant, and often unaddressed, problem. PTBID has been related to neurobiological changes resulting directly from the injury, and psychosocial factors related to life changes incurred by the injury or a combination of both (Fann, Hart, \& Schomer, 2009; Lange, Iverson, \& Rose, 2011; Seel, Macciocchi, \& Kreutzer, 2010). Understand- ing the complexity of the causal factors of PTBID has important implications for the development and implementation of interventions targeting depression in individuals with TBI.

The objective of this paper is to provide a summary of the current state of intervention research on PTBID, with the intention of highlighting the gaps in the existing literature and providing guidelines for future research. Several systematic reviews have been conducted examining the level of evidence across a broad spectrum of PTBID interventions, including pharmacological, psychosocial and alternative treatments. The main conclusion of the systematic reviews is

Address for correspondence: Theodore Tsaousides, PhD, ABPP, Assistant Professor/Director of Postdoctoral Fellowship, Department of Rehabilitation Medicine, Icahn School of Medicine at Mount Sinai, One Gustave L. Levy Place, Box 1240, New York, New York 10029, USA.

E-mail: theodore.tsaousides@mountsinai.org 
unequivocal: more research is needed. Published clinical trials either lack methodological rigour or have produced mixed findings (Alderfer, Arciniegas, \& Silver, 2005; Fann, Hart, \& Schomer, 2009; Guillamondegui et al., 2011). While evidencebased clinical and research guidelines have been provided in these reviews, only a minority of studies have been published in the past few years to advance our knowledge about treatment for PTBID further. We conducted a narrative review, focusing primarily on the nature of the interventions delivered, the eligibility criteria for inclusion in the studies, and the outcome measures on which conclusions for practice guidelines are based, because an additional systematic review, given the scarcity of recently published intervention studies, would be premature.

Our search strategy included examination of results in two databases (PubMed Medline ${ }^{\circledR}$ and PsycINFO $^{\circledR}$ ) for studies published on PTBID between 2000 and 2012, hand-searching of reference lists of systematic and other published or unpublished (e.g., grant proposals) reviews and papers on PTBID since 2000. We excluded studies that included participants younger than 16 years of age and studies that did not specify that the sample included individuals with TBI.

\section{Prevalence Rates of PTBID and Measurement Issues}

One of the main issues contributing to the inconclusive existing evidence is measurement of PTBID. Variability in measurement has not only resulted in sampling inconsistencies across studies and difficulties in comparing outcomes, but also in wide variability in estimating the prevalence of PTBID. Reported rates of PTBID over the past decade range between 12 and $60 \%$ (Ashman et al., 2004; Bombardier et al., 2010; Bryant et al., 2010; Dikmen, Bombardier, Machamer, Fann, \& Temkin, 2004; Fann et al., 2004; Glenn, O'Neil-Pirozzi, Goldstein, Burke, \& Jacob, 2001; Hart et al., 2011; Hoge et al., 2008; Holsinger et al., 2002; Jorge \& Starkstein, 2005; Koponen et al., 2002; Koponen, Taiminen, Hiekkanen, \& Tenovuo, 2011; Kreutzer, Seel, \& Gourley, 2001; Rapoport, McCullagh, Streiner, \& Feinstein, 2003a; Seel \& Kreutzer, 2003; Vanderploeg, Curtiss, Luis, \& Salazar, 2007; Whelan-Goodinson, Ponsford, Johnston, \& Grant, 2009). Figure 1 presents studies that were published after 2000, that included assessment and estimation of PTBID rates. These rates of PTBID are considerably higher than rates of depression in the general population, which range between 6 and 12\% (Keller et al., 1992; Kessler, Chiu, Demler, Merikangas, \& Walters, 2005; Mulder,
Joyce, Frampton, Luty, \& Sullivan, 2006; Pettit, Lewinsohn, \& Joiner, 2006), further highlighting the gravity of the problem for individuals with TBI.

The unusually wide range in prevalence raises questions about the appropriate diagnosis and identification of PTBID. A closer examination of studies reporting PTBID rates reveals that the variability may be attributed to differences in study design, including selection of screening and diagnostic instruments, symptom overlap between depression and TBI, injury severity, and duration of the TBI.

\section{Screening Instruments}

A variety of instruments has been used to assess the prevalence of PTBID. Among the most common diagnostic tools is the Structured Clinical Interview for Diagnostic and Statistical Manual (DSM) Disorders, which follows the diagnostic criteria of the American Psychiatric Association DSM (American Psychiatric Association, 2000), and is considered the gold standard for the diagnosis of depression in clinical and research settings (Ashman et al., 2004; Jorge \& Starkstein, 2005; Rapoport, et al., 2003a, 2003b; Seel et al., 2010; Whelan-Goodinson et al., 2009). Other similar instruments used to assess the epidemiology of PTBID that also require a clinical interview by a trained professional to render a diagnosis include the Diagnostic Interview Schedule (DIS) (Robins, Helzer, Croughan, \& Ratcliff, 1981), the Mini Neuropsychiatric Interview (MINI) (Sheehan et al., 1998), the Schedule for Clinical Assessment Interview (SCAN) (Wing et al., 1990), and the Hamilton Rating Scale for Depression (HAMD), (Hamilton, 1960). In addition to structured and unstructured clinical interviews, frequently used self-report measures that assess PTBID include the Beck Depression Inventory (BDI-II) (Beck, Steer, \& Brown, 1996), the Center for Epidemiological Studies Depression Scale (CESD) (Radloff, 1977), the PHQ-9 (Kroenke, Spitzer, \& Williams, 2001), and the Neurobehavioral Functioning Index (NFI) (Kreutzer, Marwitz, Seel, \& Serio, 1996). However, even among studies using the same instruments, different rates of PTBID are yielded, suggesting that an inconsistency in instrumentation may only account for some of the variability in estimates of PTBID.

\section{Diagnosis}

Differential diagnosis of depression from TBI symptoms may be a major factor in the variability in PTBID rates, particularly in individuals with a mild TBI (mTBI). Lange et al. (2011) identified four factors that complicate 


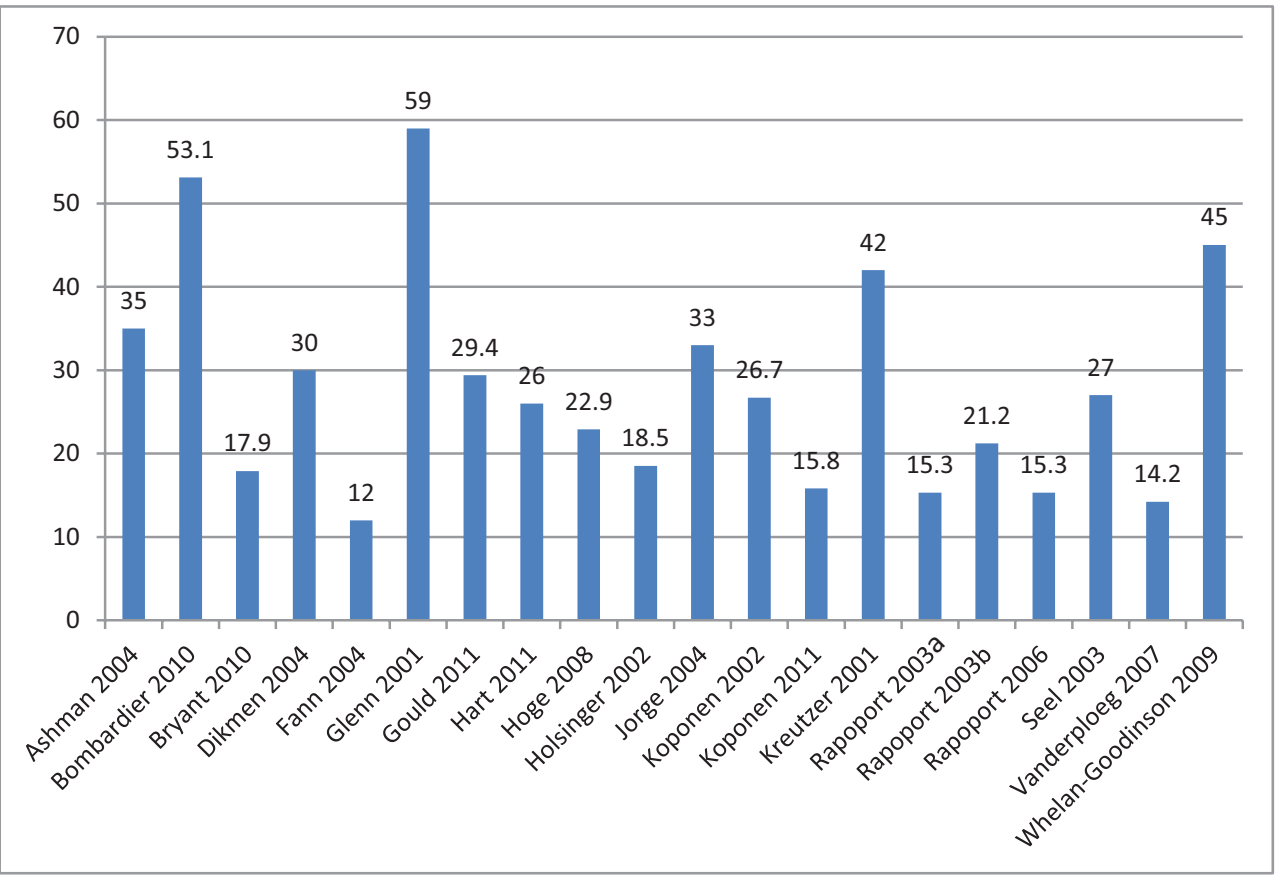

\section{FIGURE 1}

(Colour online) Empirically reported rates of depression following TBI, 2002-201 1 (numbers represent percentages; references cited by first author only).

differentiating depression from mTBI: (1) the diagnostic symptoms of depression are similar to the symptoms of post-concussion syndrome (PCS), which makes it difficult to assess the aetiology of symptom endorsement (i.e., a person with TBI may report depression-like symptoms but not suffer from depression); (2) depressive symptoms may be related to conditions comorbid with mTBI (e.g., post-traumatic stress disorder, substance abuse), making determination of causality for the depressive symptoms challenging; (3) baseline rates of depression in the general population are relatively high, making it difficult to determine whether the onset of depression was related to TBI or whether depression would occur regardless of whether a TBI was sustained; and (4) individuals with depression (and no TBI) present with similar cognitive complaints as individuals with mTBI, which implies that a person with depression would meet criteria for mTBI, even in the absence of brain injury. Anosognosia, or lack of awareness of one's TBI symptoms and functional impact, is an additional problem that may obscure the diagnosis of depression, leading to a false-negative diagnosis (Seel \& Kreutzer, 2003). Therefore, when studies use symptom report to assess depression among individuals with TBI there may be inflation or deflation of the true rates of depression.

While most of the cited studies report rates for major depressive disorder (MDD), some studies include other diagnoses of depression, including minor depression, dysthymia or unspecified depressive disorder. Hart et al. (2011) addressed the concept of minor depression and the impact on psychosocial functioning following TBI. Minor depression is qualitatively different from MDD, and thus introduces yet another dimension in terms of assessment of depression in a clinical setting. Additionally, while most studies operationalise depression on a clinical criterion, other studies use a cut-off score on a scale (e.g., BDI, HAMD) to estimate depression rates.

\section{Injury Severity and Duration}

Other factors accounting for the variability in PTBID rates across studies may be the severity and time since injury. Generally, rates of PTBID are lower among individuals with mild TBI (e.g., Bryant et al., 2010; Hoge et al., 2008; 
Vanderploeg et al., 2007), and increase as the proportion of those with moderate and severe injuries increases in the sample (e.g., Bombardier et al., 2010; Dikmen et al., 2004; Whelan-Goodinson et al., 2009). In addition, other factors to consider include the timing of the assessment for PTBID relevant to the duration of the injury. Some studies include individuals within the first year of injury (e.g., Bombardier et al., 2010; Bryant et al., 2010), while other studies have included samples with longer TBI (e.g., Ashman et al., 2004; Dikmen et al., 2004), with some evidence supporting the impression that PTBID rates decrease the longer the time since injury.

\section{Interventions}

In contrast to the plethora of published studies addressing the high prevalence of depression among individuals with TBI and the impact on various aspects of functioning, studies investigating the efficacy of interventions to reduce depression are scarce. The evidence regarding appropriate treatment for PTBID remains insufficient, making the development of treatment guidelines rely more on clinical observations and less on empirical support. In the general population, the efficacy of both pharmacological and non-pharmacological interventions targeting depression has been demonstrated repeatedly (Cuijpers, van Straten, Warmerdam, \& Andersson, 2008; Li, Frye, \& Shelton, 2012; Taylor et al., 2011). Despite the increased awareness of the prevalence of depression, the evidence regarding treatment of depression in individuals with TBI remains limited and inconclusive.

Several reviews have been conducted to assess the level of evidence for existing depression interventions following TBI (e.g., Alderfer et al., 2005; Fann et al., 2009; Guillamondegui et al., 2011; Rapoport, 2012). Fann et al. (2009) conducted a systematic review and identified 27 peer-reviewed intervention studies that met the following inclusion criteria: published after 1980, written in English, depression/depressive symptoms were primary or secondary outcome, and including only adults with TBI or reporting separately on adults with TBI. They classified these studies into pharmacological interventions (13), other biomedical interventions (6), and psychotherapeutic and rehabilitation interventions (8). Using criteria set forth by the American Academy of Neurology (AAN) to classify these studies, Fann et al. identified 2 Class I studies, 4 Class II studies and 3 Class III studies. The remaining 18 studies were classified as Class IV, suggesting that the majority of studies reporting on interventions for depression do not demonstrate sufficient methodological rigour.
A more extensive systematic review on PTBID was conducted by the Vanderbilt Evidence-based Practice Center (Guillamondegui et al., 2011). The inclusion criteria for this review were: study population was adults over 16 years of age, living in the developed world, diagnosed with depression following a TBI sustained as adults, English language, and the study designs included randomised controlled trials (RCTs), cohorts with comparison, case control or case series studies. The review resulted in 115 publications that addressed issues of prevalence, screening and treatment. While Fann et al. (2009) identified and classified 27 intervention studies, Guillamondegui et al. identified only two studies that met criteria for inclusion as interventions targeting PTBID specifically, both of which were pharmacological studies and are described further below (Ashman et al., 2009; Rapoport et al., 2008).

Another recent systematic review that focused only on non-pharmacological interventions for PTBID yielded 19 studies reporting outcomes on a depression measure (Gertler, Tate, \& Cameron, 2012). Gertler et al. reported that only four of the studies in the review involved a behavioural intervention designed to target depression specifically: a telephone counselling study (Bombardier et al., 2009), two physical activity studies (Driver \& Ede, 2009; Hoffman et al., 2010), and an individual psychotherapy case study (Ownsworth, 2005).

Guided by the available reviews, we have classified intervention studies that have included depression as an outcome measure into pharmacological, biomedical and behavioural (see Figure 2). Pharmacological studies include those that involve solely the administration of medication. Biomedical interventions include those involving a physiological intervention other than drugs, which, nevertheless, does not require the patient's active participation. Finally, behavioural interventions include non-pharmacological interventions, which require the patient's active participation.

\section{Pharmacological Interventions}

Pharmacological interventions include administration of typical antidepressant medication. Studies with TBI samples have included trials of tricyclic antidepressants (TCA), monoamine oxidase inhibitors (MAOI) and, more recently, selective serotonin reuptake inhibitors (SSRIs, including sertraline, fluoxetine, paroxetine and citalopram), as well as other agents (e.g., psychostimulants, cholinesterase inhibitors and anticonvulsants) (Alderfer et al., 2005; Fann et al., 2004).

In their review of treatments for depression, Alderfer et al. (2005) provide recommendations 
based on the existing but limited literature. They recommend SSRIs as the first-line pharmacological treatment for PTBID, and indicate that, in addition to a reduction in depression symptoms, these agents have also been shown to reduce irritability, anxiety, aggression and impulsivity in individuals with TBI. Among the SSRIs, sertraline and fluoxetine have been used most frequently in research studies, while only limited reports exist on the efficacy of paroxetine and citalopram. Older studies have included TCAs (Dinan \& Mobayed, 1992; Saran, 1985; Wroblewski, Joseph, \& Cornblatt, 1996) and MAOIs (Newburn et al., 1999), all with inconclusive findings. Alderfer et al. provide pharmacological agent and dosage recommendations specifically tailored to TBI, taking into account the susceptibility of individuals with TBI to the adverse side effects. Nevertheless, they state that these recommendations are guided more by clinical experience than by empirical evidence.

The lack of methodologically sound clinical trials is evident in these systematic reviews, which have identified only two studies that were prospectively designed to test a treatment for PTBID (Guillamondegui et al., 2011), a Class I (as per Fann et al., 2009) double-blind placebo controlled trial of sertraline (Ashman et al., 2009), and a Class IV (as per Fann et al., 2009) open-label trial of citalopram (Rapoport et al., 2008).

Ashman et al. (2009) tested the efficacy of sertraline on PTBID. To be included, participants with TBI had to meet DSM-IV diagnostic criteria for Major Depressive Disorder and have a score of 18 or higher on the Hamilton Depression Scale (HAMD). Participants were excluded from the study if they had been on an antidepressant and/or were currently receiving psychotherapy. Dosage ranged from $25 \mathrm{mg}$ to $200 \mathrm{mg}$, based on clinical response and tolerance. Forty-one participants completed the 10-week intervention. Treatment response was defined as a $50 \%$ reduction in posttreatment HAMD scores. There were no significant group differences, although approximately $59 \%$ and $32 \%$ of participants in the sertraline and placebo groups, respectively, responded positively to treatment.

Rapoport et al. (2008) conducted an open-label trial of citalopram. Their study included 54 patients with TBI who met DSM-IV diagnostic criteria for MDD. Response to treatment was defined as a reduction of $50 \%$ or more on the post-treatment HAMD, and remission was defined as a score of 7 or less on the HAMD. While initially planned as a 6-week trial, due to the low rate of response (27.7\% responded and $24.1 \%$ remitted), the trial was extended to 10 weeks. At the 10 -week assessment, out of 26 participants, $46.2 \%$ responded and
$26.9 \%$ remitted. While higher response and remission rates were observed in those who completed the 10-week regimen compared to those who completed the 6-week regimen, the difference was not significant. In a subsequent follow-up study, 21 of the participants who met criteria for remission were randomly assigned to either continue receiving citalopram or switched to placebo and were followed for an additional 40 weeks (Rapoport et al., 2010). At 40 weeks there were no significant differences between the citalopram and placebo groups in terms of the rate of, and time to, relapse. A relatively high relapse rate was found in both groups (50\% at 24.8 weeks, and $54.5 \%$ in 22.3 weeks, respectively).

Fann et al. (2009) identified a Class II pharmacological study in their systematic review. This was an RCT by Lee et al. (2005), which demonstrated the superiority of methylphenidate and sertraline over placebo in reducing HAMD scores, but not BDI scores, among 30 participants with TBI who met DSM-IV diagnostic criteria for MDD.

Of the remaining studies reviewed by Fann et al. (2009), 2 were classified as Class III and 9 as Class IV. The lack of methodological rigour notwithstanding, the results from these studies remain inconclusive, thus posing challenges to the clinical decision making of treating clinicians. Nevertheless, the majority of drug trials reviewed have been designed prospectively to evaluate the efficacy of the pharmacological agent, and in most cases established clear inclusion criteria to determine presence of depression and eligibility for participation, by using the DSM-III or DSM-IV to establish a diagnosis of major depression (Ashman et al., 2009; Dinan \& Mobayed, 1992; Fann, Uomoto, \& Katon, 2000; Kanetani, Kimura, \& Endo, 2003; Lee et al., 2005; Newburn et al., 1999; Perino, Rago, Cicolini, Torta, \& Monaco, 2001; Rapoport et al., 2008; Saran, 1985; Turner-Stokes, Hassan, Pierce, \& Clegg, 2002; Wroblewski et al., 1996). In addition, these studies have used uniform outcome measures (most frequently the HAMD, followed by the BDI), which facilitates comparison across studies and meta-analyses. This is in contrast to the most frequently cited behavioural interventions.

\section{Other Biomedical Interventions}

In addition to pharmacological studies targeting neurochemical functioning, several studies have explored the effectiveness of other types of biomedical interventions in reducing PTBID. These interventions have shown promising results in other clinical populations with depression but no brain injury. Fann et al. (2009), identified two 
studies that used electroconvulsive treatment (ECT) (Kant, Coffey, \& Bogyi, 1999; Martino, Krysko, Petrides, Tobias, \& Kellner, 2008), two studies of transcerebral magnetic field treatment (TCM) (Baker-Price \& Persinger, 1996, 2003), a study of electroencephalography-based (EEG) biofeedback (Schoenberger, Shif, Esty, Ochs, \& Matheis, 2001), and a case study of acupuncture following TBI (Donnellan, 2006).

Kant et al. (1999) conducted a case series study of 11 participants with TBI and depression treated with ECT. Upon enrolment, four participants were diagnosed with MDD, five with mood disorder secondary to closed head injury, one with chronic delirium, and one with delusional disorder. Eight patients $(72.7 \%)$ were classified as responders, two as partial responders and one participant as nonresponder. Only one of the four participants diagnosed with MDD responded to treatment. Martino et al. (2008) published a case study to demonstrate reduction in persistent depression following ECT in a patient with TBI and a history of depression. The patient had been diagnosed with depression prior to the injury and continued to experience symptoms that required hospitalisation for depression post-injury. The patient did not respond well to a variety of medications and relapsed after two courses of ECT treatment. Schoenberger et al. (2001) reported mood improvement on the BDI after 25 sessions of EEG biofeedback. However, there were no depression eligibility criteria in this study, and therefore conclusions about its effectiveness in treating depression are limited. Similarly, depression was not an inclusion criterion for a case report of the effectiveness of acupuncture for pain, and even though the author reported mood improvement based on patient self-report, this improvement was not reflected in the standardised outcome measures.

Finally, in two studies, Baker-Price and Persinger $(1996,2003)$ noted significant mood improvements on the BDI following TCM, for 4 and 14 patients with TBI, respectively. However, the eligibility criteria were self-report of depressive symptoms and/or (unspecified) prior diagnosis by a physician (unclear whether formal diagnostic criteria were used). More recently, Fitzgerald et al. (2011) published a case study of PTBID treatment using transcranial magnetic stimulation (TMS), on a female patient with mild to moderate TBI and persistent PTBID. They reported a 50\% reduction in depression symptoms on a clinical rating scale. Although promising, the findings of these studies should be viewed with caution, because of their methodological limitations. More research is necessary to determine whether such treatments constitute a viable alternative treatment for PTBID.

\section{Behavioural Interventions}

Reviews of behavioural interventions for the treatment of PTBID have included an assortment of treatments that are theoretically and clinically dissimilar, making comparison across studies and development of guidelines for the treatment of PTBID challenging. The existing reviews of behavioural interventions have included studies that cover a disparate group of interventions (ranging from aquatic exercise to individual psychotherapy) that use mixed modalities of treatment (one-on-one treatment vs. comprehensive outreach neurorehabilitation), have a broad range of treatment targets (e.g., depression, emotional adjustment, independence, coping skills), and do not always specify inclusion/exclusion criteria for depression. More importantly, the majority of the studies included in the reviews below were not designed prospectively to treat depression, even though they provide some data about changes in mood following an intervention that was originally designed to address a target other than depression.

One common characteristic that differentiates interventions in this category from pharmacological and biomedical treatment is the active role of the patient in the treatment. To facilitate the review and attempt to disentangle study findings for these behavioural interventions for PTBID, in order to generate conclusions for clinical practice, the treatments have been subdivided based on theoretical similarities: physical activity, mindfulnessbased and psychosocial interventions. Psychosocial interventions could be further subdivided into individual face-to-face (FTF) psychological treatments, individual non-FTF psychological treatments, group coping skills interventions and comprehensive neurorehabilitation (Figure 2).

In terms of the level of evidence, in their published review, Fann et al. (2009) identified 8 studies reporting outcomes on mood following a behavioural intervention: 1 Class I study (Powell, Heslin, \& Greenwood, 2002), 2 Class II studies (McMillan, Robertson, Brock, \& Chorlton, 2002; Tiersky et al., 2005) and 5 Class IV. Again the lack of methodological rigour renders the state of the evidence weak. Given that all but one of these studies were not designed to target depression directly, a great deal more research is needed to identify appropriate and effective interventions for PTBID to promote the establishment of treatment guidelines for post-TBI depression.

\section{Physical Activity}

Two studies have reported findings on mood for individuals with TBI following a physical activity intervention. Specifically, Driver and Ede 


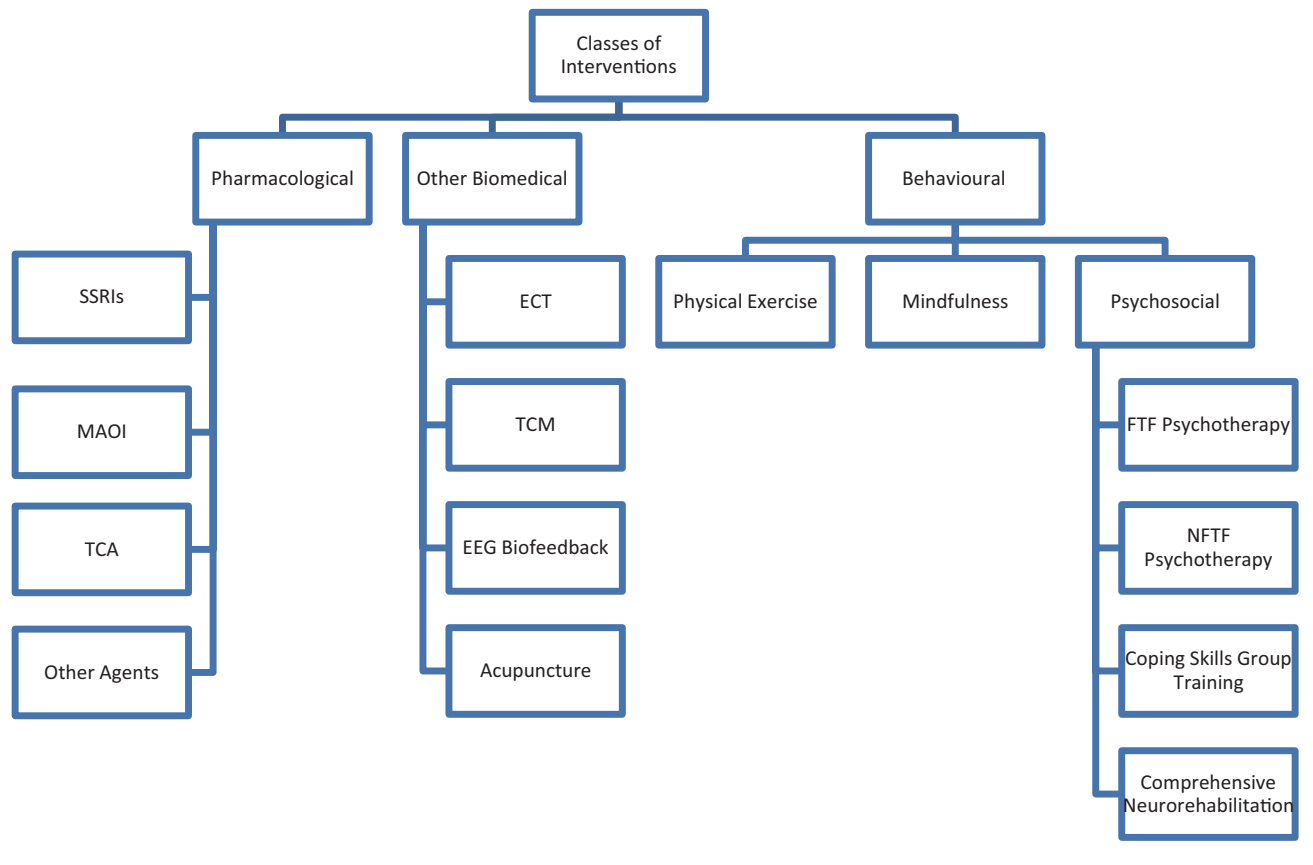

\section{FIGURE 2}

(Colour online) Classes of intervention studies for post-traumatic brain injury depression. SSRI, selective serotonin reuptake inhibitors; MAOI, monoamine oxidase inhibitors; TCA, tricyclic antidepressants; ECT, electroconvulsive treatment; TCM, transcerebral magnetic field treatment; EEG, electroencephalography-based; FTF, face-to-face; NFTF, non-face-to-face.

(2009) conducted an RCT to examine the effects of aquatic exercise on mood states. Sixteen participants were randomly assigned to either an 8week (24-session) aquatic fitness programme or vocational rehabilitation treatment of equal duration and intensity. Significant between-group and within-group differences were found, with large effect sizes detected in pre-post depression scores in the experimental group, with no change in the control group.

Hoffman et al. (2010) conducted a wait-list control RCT to examine the impact of aerobic exercise on severity of depression. Participants in the immediate exercise group received 10 weekly sessions of aerobic exercise, and were encouraged to exercise additionally at home during the week. The wait-list control group was given no specific instructions regarding physical activity. The authors reported no significant group differences on the BDI, but found an effect for amount of activity (participants in either group who exercised for longer overall had better mood outcomes).

While the results of these studies are promising, especially with respect to the relationship between amount of activity and mood, in neither study was a diagnosis of depression an eligibility criterion for enrolment. Hoffman et al. (2010) enrolled participants who scored in the mild depressive range on the PHQ-9, while Driver and Ede (2009) did not include level of mood symptoms in their inclusion criteria.

\section{Mindfulness Training}

McMillan et al. (2002) assessed the efficacy of attentional control training (ACT) on attention and mood. Participants were assigned to one of three conditions: ACT, physical exercise (PE) or no treatment. The ACT group received five 45-minute supervised sessions of mindfulness training and instructions to practise daily on their own during the 4-week treatment period. The PE group received a similar number of supervised sessions of $\mathrm{PE}$ and instructions to practise PE on their own. The control group received no intervention and only completed the outcome assessments. Mood was assessed pre- and post-treatment with the Hospital Anxiety and Depression Scales (Zigmond \& Snaith, 1983). However, there were no predetermined cut-off scores on these measures for 
inclusion in the study. The authors found no between- or within-group differences in depression or anxiety.

Bedard et al. (2003) conducted a pilot study to evaluate a mindfulness-based stress reduction intervention to improve quality of life. The intervention consisted of 12 weekly group sessions, using a popular mindfulness-based stress reduction technique. Significant within-group changes were found on the cognitive-affective subscale of the BDI-II but only marginal differences on the total and the somatic scales. Interestingly, the authors excluded from their sample individuals with a major DSM-IV mental illness, which implies that individuals with a diagnosable mood disorder were not included in the study. Therefore, whether the intervention is a suitable alternative for the treatment of PTBID remains unknown.

In 2012, Bedard et al. reported findings on a mindfulness-based intervention, specifically for depression. The authors utilised a protocol that combined mindfulness training with elements of cognitive therapy. They included participants who showed 'evidence of clinical depression' using the DSM-IV. In their follow-up description, however, it is unclear whether the participants met DSM-IV diagnostic criteria for MDD or other depressive disorder at the time of enrolment in the study. Instead, the presence of depression was established through chart reviews of previous DSM-IV interviews and diagnoses, and assessment by a physician to establish that participants had 'clear signs of depression'. While no control group was involved, the authors reported significant reduction in BDI, PHQ-9 and HADS-Depression scores, after 8 weekly 90 -minute sessions of mindfulnessbased cognitive therapy. Further DSM-IV interviews were not conducted at the end of treatment.

\section{Psychosocial Interventions}

Individual FTF psychological interventions. Among the individual psychotherapy interventions, cognitive behavioural therapy (CBT) is one of the most empirically supported treatments for depression in the general population (Butler, Chapman, Forman, \& Beck, 2006). CBT techniques are also particularly well-suited for engaging individuals with TBI in treatment (Anson \& Ponsford, 2006; Doering \& Exner, 2011). The efficacy of CBT following TBI has been demonstrated in studies targeting management of PCS (Mittenberg, Tremont, Zielinski, Fichera, \& Rayls, 1996), social anxiety (Hodgson, McDonald, Tate, \& Gertler, 2005), insomnia (Ouellet \& Morin, 2007), anger management (Medd \& Tate, 2000), hopelessness
(Simpson, Tate, Whiting, \& Cotter, 2011) and management of headaches (Gurr \& Coetzer, 2005).

While elements of CBT have been incorporated in interventions targeting emotional adjustment and psychological functioning (e.g., Anson \& Ponsford, 2006; Backhaus, Ibarra, Klyce, Trexler, \& Malec, 2010; Tiersky et al., 2005), the efficacy of individual CBT for depression has not been examined to date. The only prospective study that was designed to specifically examine the efficacy of individual CBT on PTBID was a RCT conducted by Ashman et al. (2011). Participants were randomised into either a 16-session treatment of CBT $(N=36)$ or supportive psychotherapy $(N=33)$. The remission rates for depression were significant for both individuals in each of the treatments, $57 \%$ for CBT and $40 \%$ for supportive psychotherapy; however, there was no significant between-group difference, largely due to small sample size.

Ownsworth (2005) reported on a case study, which involved treatment of an individual with TBI to address the effects of defensive denial on emotional functioning. Although the participant's pre-treatment scores on a measure of depression and anxiety were in the severe range, it is unclear whether scores on this scale were used as an inclusion criterion. In fact, the author states that the most consistent DSM-IV diagnosis for the participant was Social Anxiety Disorder with depressive symptoms secondary to that diagnosis. Hence, despite reporting significant improvements in mood post-treatment, the intervention described in this study was not delivered to address PTBID specifically.

Tiersky et al. (2005) assessed the efficacy of a neuropsychological rehabilitation programme combining psychotherapy and cognitive remediation for individuals with mild TBI. Participants were assigned either to the treatment group or a wait-list control group. One of the inclusion criteria was 'complaints of emotional distress' (p. 1566) on the Symptom Checklist-90 Revised (Derogatis, 1994). The intervention included three weekly 50-minute individual sessions of CBT and three weekly 50-minute individual sessions of cognitive remediation. Reduction in overall emotional distress was noted, and improvement on the subscales for depression and anxiety were also evident in the immediate treatment group. Given that the inclusion criterion for mood was not specific to depression nor was a diagnosis of depression required, conclusions about the efficacy of this intervention for the treatment of PTBID are limited. In addition, it is unclear whether this treatment would qualify as intensive individual psychotherapy with dual focus, or would be better described as comprehensive neurorehabilitation, given that 
participation required inclusion of cognitive and affective symptoms.

Finally, Gurr \& Coetzer (2005) conducted a study on the effectiveness of CBT for the management of post-concussive headaches. The intervention consisted of six biweekly individual CBT sessions. While there were positive findings reported in terms of headache management, findings on emotional well-being were mixed. Although this study has been included in the two systematic reviews (Fann et al., 2009; Gertler et al., 2012), it is not a study that provides insight about the treatment of PTBID.

\section{Individual non-FTF psychological interventions.} In addition to FTF interventions, studies have reported on mood outcomes using different treatment modalities. Bombardier et al. (2009) reported improvements in depression across three outcome measures in the experimental group that received a telephone counselling intervention compared to the control group that received care-as-usual. The Structured Telephone Intervention (STI) consisted of seven regularly scheduled 30-45 telephone sessions over a period of 9 months. The sessions included follow-up on previously stated concerns, addressing new concerns and providing interventions (see Bell et al., 2005). Nevertheless, this study was originally designed to test the effectiveness of the phone counselling intervention across a variety of behavioural and functional outcomes (Bell et al., 2005), and therefore was not designed specifically to ameliorate depression, nor did it prospectively recruit individuals with TBI and a diagnosis of depressive disorder. Moreover, in a subsequent RCT (Bell et al., 2011) the authors failed to replicate the earlier findings.

Topolovec-Vranic et al. (2010) evaluated the effectiveness of an online CBT programme for PTBID using a pre-post design with no control group. Twenty-one participants with TBI were identified who met criteria for moderate depression on the PHQ-9. The intervention included six weekly internet-based, self-administered sessions designed to address symptoms of depression, accompanied by six weekly phone calls to ascertain use of the website. While only 13 participants completed all outcome assessments, significant improvements in mood were noted both at the end of treatment as well as at the 12-week follow-up assessment.

Group coping skills interventions. Another type of intervention that has been associated with improvements in mood and emotional adjustment post-TBI includes CBT-based group interventions with emphasis on developing coping skills. Two studies were identified that describe such interven- tions. The first study is an evaluation of a coping skills group using a wait-list control design (Anson \& Ponsford, 2006), which involved 36 individuals with TBI, who did not, however, present with clinical levels of emotional maladjustment. The intervention included 10 biweekly 90 -minute group sessions, over 5 weeks. While there was evidence of improvement in terms of adaptive coping, the intervention did not result in changes in mood post-treatment. Backhaus et al. (2010) conducted a randomised wait-list control design to assess the efficacy of a coping skills training intervention on psychological functioning and selfefficacy. Twenty participants were randomly assigned to either a treatment or a control group. Participants in the treatment group received 122 hour sessions of psychoeducation, psychotherapy, stress-management and problem-solving. The novelty of the intervention was that caregivers were included in the treatment groups, although outcome measures were obtained only for the participants. While participants in the treatment group demonstrated significantly higher perceived self-efficacy at the end of treatment, levels of psychological distress did not differ between the two groups at either the end of treatment or the 3-month follow-up assessment. Given that neither of the two studies established eligibility criteria for depression, the effectiveness of coping skills training on treating PTBID remains unknown.

Comprehensive neurorehabilitation. While not designed to treat depression, several studies have reported on mood outcomes following participation in comprehensive rehabilitation programmes. Powell et al. (2002) conducted a study to examine the effects of a community-based multidisciplinary programme on independence in activities of daily living, social participation and psychological wellbeing following severe TBI. Participants received either an individualised, home-based, 2- to 6-hour weekly treatment programme for an average of 28 weeks (physical/occupational therapy, psychology) or 1-hour information session about local and national resources. No differences were detected between the experimental and control groups in terms of changes in depression and anxiety postintervention as measured by the HADS. Nevertheless, participants were included in the study, regardless of their scores on the mood scales, and in fact the majority of participants scored within the normal range on both.

Finally, Svendsen, Teasdale, \& Pinner (2004) reported on a retrospective study comparing responses of individuals who had completed a comprehensive neuropsychological rehabilitation program pre- and post-treatment on a comprehensive 
symptom checklist (EBIQ). The programme consisted of daily participation in group treatment for 4 months, with patient contact and monitoring for an additional 8 months after discharge. The authors reported improvements on the depression scale for the TBI subsample. However, the study was not designed prospectively to target depression, and depression criteria were not used to established eligibility for participation.

\section{Implications for Future Research}

Despite the high rates of depression in this population, and the potential impact of emotional maladjustment on psychosocial functioning and recovery, only $44 \%$ of individuals with TBI diagnosed with depression receive some type of treatment for depression (Bombardier et al., 2010). Depression is one of the most common psychiatric conditions in the general population, and one of the leading causes of disability in the US. While wellestablished, empirically supported treatments exist for the treatment of depression, the state of the evidence for the treatment of PTBID is far from ideal. Given the variability in the methodological approaches implemented to identify the prevalence of depression, two of the main considerations in developing and testing interventions for PTBID include targeting interventions appropriately to patient characteristics, and selecting appropriate screening and outcome measures. In terms of targeting or adapting interventions appropriately, one important factor to consider is injury severity. The degree to which a person experiences physical or cognitive limitations impacts treatment delivery and influences treatment outcomes. A person with limited attention may have difficulty benefiting from a 45-minute individual psychotherapy session, while another person with disinhibition and agitation may be disruptive in a group setting, thus undermining the effect of treatment for themselves and others. Another factor to consider is the source of the sample. Soldiers on active duty may have different responses to treatment from older adults in retirement or college athletes. Assessing and addressing factors that maintain depression may be instrumental. For example, given the positive influence of social support on mood, it is likely that individuals with a supportive network will respond to treatment better or faster, than those experiencing social isolation. Assessing prior psychiatric history may provide useful insight, in terms of one's disposition as well as previous responses to treatment. The chronicity of injury may also influence how individuals respond to treatment. More recently injured individuals may exhibit lack of awareness of deficits or be preoccupied with recovery of physical injuries and thus overlook the need for pharmacological or psychological treatment for mood. More remotely injured individuals may have developed chronic maladaptive coping patterns more intransigent to change, or may have experienced the psychosocial consequences of the injury longer and thus experience more pessimism and hopelessness. An additional issue to consider is whether early intervention among those who are assessed to be at risk for developing PTBID, based on studies that have identified risk factors for PTBID (Ashman et al., 2004; Bombardier et al., 2010), might prevent the subsequent development of clinically significantly levels of PTBID.

In terms of instrument selection, it is important to determine clear depression-related inclusion criteria in designing studies, to ensure that treatment effects are not diluted because of the low threshold for participation in treatment. Establishing a priori criteria for improvement is essential. Using the same measure pre- and post-treatment (whether interview-based diagnosis or cut-off score on a self-report measure) increases the validity of the findings. Appropriate measure selection enables appropriate stratification by symptom severity or diagnosis. Therefore, people with minor depression could be identified early on and analysed separately from those with major depression; or people with higher suicidal ideation could receive a modified intervention compared to those without suicidal ideation. Finally, uniformity in the selection of screening and outcome measures will facilitate the examination of the existing evidence through systematic reviews and meta-analyses and will promote the development of clear guidelines for both clinicians and researchers (see Table 1 for a summary of recommendations).

\section{Conclusion}

The high rates of depression following TBI have been clearly defined in the literature for many decades, and within the past decade clinical researchers have been designing interventions to address PTBID. However, the results of many of these empirical treatment studies have been inconclusive, often due to small sample sizes but also because of large variability in methodological approaches, including patient and injury characteristics, and screening and outcome measures. The field is in need of large, appropriately controlled pharmacological, biomedical, psychotherapeutic and/or rehabilitation interventions, that can support comparisons of different treatments and account for premorbid psychiatric history, including treatment history, injury variables (e.g., 


\section{TABLE 1}

Recommendations for Designing Interventions for PTBID

Measurement

- Select measures with good psychometric properties and appropriately normed for individuals with TBI

- Consider diagnostic tools that rely on clinical interview, to reduce cognitive demands, assess functional impact, and differentiate between depression and $\mathrm{mTBI}$ symptoms

- Determine a priori an empirically guided, clinically significant cut-off score

- Include the screening measure as an outcome measure to determine efficacy

Patient characteristics

- Include participants who meet criteria for clinically significant levels of depression (or subtype)

- Stratify sample by depression subtype, if necessary (e.g., Major Depressive Disorder, Dysthymic Disorder, minor depression)

- Stratify sample by injury severity and time since injury

- Assess current psychiatric history to rule out co-existing disorders with similar symptoms

- Assess prior psychiatric history

- Assess prior treatment history

Intervention

- Select interventions that are theoretically or empirically expected to reduce depression

- Modify intervention appropriately to address physical and cognitive limitations

- Modify intervention to source sample (e.g., military, college students)

severity, time since injury, cognitive impairments) and psychosocial factors. The variability among individuals with TBI indicates that knowing that a person has PTBID may not be sufficient enough to identify the most appropriate treatment modality without assessing other factors associated with effective treatment for depression. Future studies need to address directly the causal nature of factors that have been identified as impacting depression following TBI, to further develop treatment modalities maximally suited to treating depression for specific subgroups of individuals with TBI.

\section{References}

Alderfer, B.S., Arciniegas, D.B., \& Silver, J.M. (2005). Treatment of depression following traumatic brain injury. Journal of Head Trauma Rehabilitation, 20(6), 544-562.

American Psychiatric Association. (2000). Diagnostic and statistical manual of mental disorders (4th ed., text revision). Washington, DC: American Psychiatric Press.

Anson, K., \& Ponsford, J. (2006). Evaluation of a coping skills group following traumatic brain injury. Brain Injury, 20(2), 167-178.

Ashman, T.A., Cantor, J.B., Gordon, W.A., Spielman, L., Flanagan, S., Ginsberg, A., ... Greenwald, B. (2009). A randomized controlled trial of sertraline for the treatment of depression in persons with traumatic brain injury. Archives of Physical Medicine and Rehabilitation, 90(5), 733-740.

Ashman, T.A., Spielman, L.A., Hibbard, M.R., Silver, J.M., Chandna, T., \& Gordon, W.A. (2004). Psychiatric challenges in the first 6 years after traumatic brain injury: Cross-sequential analyses of axis I disorders. Archives of Physical Medicine and Rehabilitation, 85(4 Suppl 2), S36-42.

Ashman, T.A., Tsaousides, T., \& Cantor, J.B. (2011). Empirically-based interventions for post-TBI depression. Presented at the American Congress of Rehabilitation Medicine, October, Atlanta, GA.

Backhaus, S.L., Ibarra, S.L., Klyce, D., Trexler, L.E., \& Malec, J.F. (2010). Brain injury coping skills group: A preventative intervention for patients with brain injury and their caregivers. Archives of Physical Medicine and Rehabilitation, 91(6), 840-848.

Baker-Price, L., \& Persinger, M.A. (2003). Intermittent burst-firing weak (1 microTesla) magnetic fields reduce psychometric depression in patients who sustained closed head injuries: A replication and electroencephalographic validation. Perceptual and Motor Skills, 96(3 Pt 1), 965-974.

Baker-Price, L.A., \& Persinger, M.A. (1996). Weak, but complex pulsed magnetic fields may reduce depression following traumatic brain injury. Perceptual and Motor Skills, 83(2), 491-498.

Beck, A.T., Steer, R., \& Brown, G. (1996). Beck depression inventory-II. San Antonio: Psychological Corporation.

Bedard, M., Felteau, M., Marshall, S., Dubois, S., Gibbons, C., Klein, R., ... Weaver, B. (2012). Mindfulness-based cognitive therapy: Benefits in reducing depression following a traumatic brain injury. Advances in Mind Body Medicine, 26, 14-20.

Bedard, M., Felteau, M., Mazmanian, D., Fedyk, K., Klein, R., Richardson, J., . . Minthorn-Biggs, M.B. (2003). Pilot evaluation of a mindfulness-based intervention to improve quality of life among individuals who sustained traumatic brain injuries. Disability and Rehabilitation, 25(13), 722-731.

Bell, K.R., Brockway, J.A., Hart, T., Whyte, J., Sherer, M., Fraser, R.T., ... Dikmen, S.S. (2011). Scheduled telephone intervention for traumatic brain injury: A multicenter randomized controlled trial. Archives of Physical Medicine and Rehabilitation, 92(10), 1552-1560.

Bell, K.R., Temkin, N.R., Esselman, P.C., Doctor, J.N., Bombardier, C.H., Fraser, R.T., ... Dikmen, S. (2005). The effect of a scheduled telephone intervention on outcome after moderate to severe 
traumatic brain injury: A randomized trial. Archives of Physical Medicine and Rehabilitation, 86(5), 851856.

Bombardier, C.H., Bell, K.R., Temkin, N.R., Fann, J.R., Hoffman, J., \& Dikmen, S. (2009). The efficacy of a scheduled telephone intervention for ameliorating depressive symptoms during the first year after traumatic brain injury. Journal of Head Trauma Rehabilitation, 24(4), 230-238.

Bombardier, C.H., Fann, J.R., Temkin, N.R., Esselman, P.C., Barber, J., \& Dikmen, S.S. (2010). Rates of major depressive disorder and clinical outcomes following traumatic brain injury. JAMA: The Journal of the American Medical Association, 303(19), 19381945.

Bryant, R.A., O'Donnell, M.L., Creamer, M., McFarlane, A.C., Clark, C.R., \& Silove, D. (2010). The psychiatric sequelae of traumatic injury. American Journal of Psychiatry, 167(3), 312-320.

Butler, A.C., Chapman, J.E., Forman, E.M., \& Beck, A.T. (2006). The empirical status of cognitive-behavioral therapy: A review of meta-analyses. Clinical Psychology Review, 26(1), 17-31.

Cuijpers, P., van Straten, A., Warmerdam, L., \& Andersson, G. (2008). Psychological treatment of depression: A meta-analytic database of randomized studies. BMC Psychiatry, 8, 36.

Derogatis, L.R. (1994). Administration, scoring and procedures manual for the SCL-90-R. Minneapolis: National Computer Systems.

Dikmen, S.S., Bombardier, C.H., Machamer, J.E., Fann, J.R., \& Temkin, N.R. (2004). Natural history of depression in traumatic brain injury. Archives of Physical Medicine and Rehabilitation, 85(9), 1457-1464.

Dinan, T.G., \& Mobayed, M. (1992). Treatment resistance of depression after head injury: A preliminary study of amitriptyline response. Acta Psychiatrica Scandinavica, 85(4), 292-294.

Doering, B., \& Exner, C. (2011). Combining neuropsychological and cognitive-behavioral approaches for treating psychological sequelae of acquired brain injury. Current Opinion in Psychiatry, 24(2), 156-161.

Donnellan, C.P. (2006). Acupuncture for central pain affecting the ribcage following traumatic brain injury and rib fractures - a case report. Acupuncture in Medicine: Journal of the British Medical Acupuncture Society, 24(3), 129-133.

Driver, S., \& Ede, A. (2009). Impact of physical activity on mood after TBI. Brain Injury, 23(3), 203-212.

Fann, J.R., Burington, B., Leonetti, A., Jaffe, K., Katon, W.J., \& Thompson, R.S. (2004). Psychiatric illness following traumatic brain injury in an adult health maintenance organization population. Archives of General Psychiatry, 61(1), 53-61.

Fann, J.R., Hart, T., \& Schomer, K.G. (2009). Treatment for depression after traumatic brain injury: A systematic review. Journal of Neurotrauma, 26(12), 2383-2402.

Fann, J.R., Uomoto, J.M., \& Katon, W.J. (2000). Sertraline in the treatment of major depression following mild traumatic brain injury. Journal of Neuropsychiatry and Clinical Neurosciences, 12(2), 226-232.

Fitzgerald, P.B., Hoy, K.E., Maller, J.J., Herring, S., Segrave, R., McQueen, S., ... Daskalakis, Z.J. (2011). Transcranial magnetic stimulation for depression after a traumatic brain injury: A case study. Journal of ECT, 27(1), 38-40.

Gertler, P., Tate, R.L., \& Cameron, I.D. (2012). Nonpharmacological interventions for depression in adults and children with traumatic brain injury. Cochrane Database of Systematic Reviews, Issue 5. Art. No.: CD009871. DOI: 0.1002/14651858. CD009871

Glenn, M.B., O’Neil-Pirozzi, T., Goldstein, R., Burke, D., \& Jacob, L. (2001). Depression amongst outpatients with traumatic brain injury. Brain Injury, 15(9), 811818.

Gould, K.R., Ponsford, J.L., Johnston, L., \& Schoenberger, M. (2001). The nature, frequency and course of psychiatric disorders the first year after traumatic brain injury: a prospective study. Psychological Medicine, 41, 2099-2109.

Guillamondegui, O.D., Montgomery, S.A., Phibbs, F.T., McPheeters, M.L., Alexander, P.T., Jerome, R.N. ... Hartmann, K.E. (2011). Traumatic brain injury and depression. Comparative Effectiveness Review No. 25. (Prepared by the Vanderbilt Evidencebased Practice Center under Contract No. 290-200710065-I.) AHRQ Publication No. 11-EHC017-EF. Rockville, MD: Agency for Healthcare Research and Quality.

Gurr, B., \& Coetzer, B.R. (2005). The effectiveness of cognitive-behavioural therapy for post-traumatic headaches. Brain Injury, 19(7), 481-491.

Hamilton, M. (1960). A rating scale for depression. Journal of Neurology, Neurosurgery, and Psychiatry, 23, 56-62.

Hart, T., Brenner, L., Clark, A.N., Bogner, J.A., Novack, T.A., Chervoneva, I., ... Arango-Lasprilla, J.C. (2011). Major and minor depression after traumatic brain injury. Archives of Physical Medicine and Rehabilitation, 92(8), 1211-1219.

Hodgson, J., McDonald, S., Tate, R., \& Gertler, P. (2005). A randomised controlled trial of a cognitivebehavioural therapy program for managing social anxiety after acquired brain injury. Brain Impairment, 6(3), 169.

Hoffman, J.M., Bell, K.R., Powell, J.M., Behr, J., Dunn, E.C., Dikmen, S., ... Bombardier, C.H. (2010). A randomized controlled trial of exercise to improve mood after traumatic brain injury. $P M \& R$ : The Journal of Injury, Function, and Rehabilitation, 2(10), 911-919.

Hoge, C.W., McGurk, D., Thomas, J.L., Cox, A.L., Engel, C.C., \& Castro, C.A. (2008). Mild traumatic brain injury in U.S. soldiers returning from iraq. New England Journal of Medicine, 358(5), 453-463.

Holsinger, T., Steffens, D.C., Phillips, C., Helms, M.J., Havlik, R.J., Breitner, J.C., . . Plassman, B.L. (2002). Head injury in early adulthood and the 
lifetime risk of depression. Archives of General Psychiatry, 59(1), 17-22.

Jorge, R.E., Robinson, R.G., Moser, D., Tateno, A., Crespo-Facorro, B., \& Arndt, S. (2004). Major depression following traumatic brain injury. Archives of General Psychiatry, 61, 42-50.

Jorge, R.E., \& Starkstein, S.E. (2005). Pathophysiologic aspects of major depression following traumatic brain injury. Journal of Head Trauma Rehabilitation, 20(6), 475-487.

Kanetani, K., Kimura, M., \& Endo, S. (2003). Therapeutic effects of milnacipran (serotonin noradrenalin reuptake inhibitor) on depression following mild and moderate traumatic brain injury. Journal of Nihon Medical School, 70(4), 313-320.

Kant, R., Coffey, C.E., \& Bogyi, A.M. (1999). Safety and efficacy of ECT in patients with head injury: A case series. Journal of Neuropsychiatry and Clinical Neurosciences, 11(1), 32-37.

Keller, M.B., Lavori, P.W., Mueller, T.I., Endicott, J., Coryell, W., Hirschfeld, R.M., ... Shea, T. (1992). Time to recovery, chronicity, and levels of psychopathology in major depression. A 5-year prospective follow-up of 431 subjects. Archives of General Psychiatry, 49(10), 809-816.

Kessler, R.C., Chiu, W.T., Demler, O., Merikangas, K.R., $\&$ Walters, E.E. (2005). Prevalence, severity, and comorbidity of 12-month DSM-IV disorders in the national comorbidity survey replication. Archives of General Psychiatry, 62(6), 617-627.

Koponen, S., Taiminen, T., Hiekkanen, H., \& Tenovuo, O. (2011). Axis I and II psychiatric disorders in patients with traumatic brain injury: A 12-month follow-up study. Brain Injury, 25(11), 1029-1034.

Koponen, S., Taiminen, T., Portin, R., Himanen, L., Isoniemi, H., Heinonen, H., ... Tenovuo, O. (2002). Axis I and II psychiatric disorders after traumatic brain injury: A 30-year follow-up study. American Journal of Psychiatry, 159(8), 1315-1321.

Kreutzer, J.S., Marwitz, J.H., Seel, R., \& Serio, C.D. (1996). Validation of a neurobehavioral functioning inventory for adults with traumatic brain injury. Archives of Physical Medicine and Rehabilitation, 77(2), 116-124.

Kreutzer, J.S., Seel, R.T., \& Gourley, E. (2001). The prevalence and symptom rates of depression after traumatic brain injury: A comprehensive examination. Brain Injury, 15(7), 563-576.

Kroenke, K., Spitzer, R.L., \& Williams, J.B. (2001). The PHQ-9: Validity of a brief depression severity measure. Journal of General Internal Medicine, 16(9), 606-613.

Lange, R.T., Iverson, G.L., \& Rose, A. (2011). Depression strongly influences postconcussion symptom reporting following mild traumatic brain injury. Journal of Head Trauma Rehabilitation, 26(2), 127-137.

Lee, H., Kim, S.W., Kim, J.M., Shin, I.S., Yang, S.J., \& Yoon, J.S. (2005). Comparing effects of methylphenidate, sertraline and placebo on neuropsychiatric sequelae in patients with traumatic brain injury. Human Psychopharmacology, 20(2), 97-104.

Li, X., Frye, M.A., \& Shelton, R.C. (2012). Review of pharmacological treatment in mood disorders and future directions for drug development. Neuropsychopharmacology, 37(1), 77-101.

Martino, C., Krysko, M., Petrides, G., Tobias, K.G., \& Kellner, C.H. (2008). Cognitive tolerability of electroconvulsive therapy in a patient with a history of traumatic brain injury. Journal of ECT, 24(1), 9295.

McMillan, T.M., Robertson, I.H., Brock, D., \& Chorlton, L. (2002). Brief mindfulness training for attentional problems after traumatic brain injury: A randomised control treatment trial. Neuropsychological Rehabilitation, 12(2), 117-125.

Medd, J., \& Tate, R.L. (2000). Evaluation of an anger management therapy programme following acquired brain injury: A preliminary study. Neuropsychological Rehabilitation, 10(2), 185-201.

Mittenberg, W., Tremont, G., Zielinski, R.E., Fichera, S., \& Rayls, K.R. (1996). Cognitive-behavioral prevention of postconcussion syndrome. Archives of Clinical Neuropsychology, 11(2), 139-145.

Mulder, R.T., Joyce, P.R., Frampton, C.M., Luty, S.E., $\&$ Sullivan, P.F. (2006). Six months of treatment for depression: Outcome and predictors of the course of illness. American Journal of Psychiatry, 163(1), 95-100.

Newburn, G., Edwards, R., Thomas, H., Collier, J., Fox, K., \& Collins, C. (1999). Moclobemide in the treatment of major depressive disorder (DSM-3) following traumatic brain injury. Brain Injury, 13(8), 637642.

Ouellet, M.C., \& Morin, C.M. (2007). Efficacy of cognitive-behavioral therapy for insomnia associated with traumatic brain injury: A single-case experimental design. Archives of Physical Medicine and Rehabilitation, 88(12), 1581-1592.

Ownsworth, T. (2005). The impact of defensive denial upon adjustment following traumatic brain injury. Neuro-Psychoanalysis, 7(1), 83-94.

Perino, C., Rago, R., Cicolini, A., Torta, R., \& Monaco, F. (2001). Mood and behavioural disorders following traumatic brain injury: Clinical evaluation and pharmacological management. Brain Injury, 15(2), 139-148.

Pettit, J.W., Lewinsohn, P.M., \& Joiner, T.E. Jr. (2006). Propagation of major depressive disorder: Relationship between first episode symptoms and recurrence. Psychiatry Research, 141(3), 271-278.

Powell, J., Heslin, J., \& Greenwood, R. (2002). Community based rehabilitation after severe traumatic brain injury: A randomised controlled trial. Journal of Neurology, Neurosurgery, and Psychiatry, 72(2), 193-202.

Radloff, L.S. (1977). The CES-D scale: A self-report depression scale for research in the general population. Applied Psychological Measurement, 1(3), 385 . 
Rapoport, M.J. (2012). Depression following traumatic brain injury: Epidemiology, risk factors and management. CNS Drugs, 26(2), 111-121.

Rapoport, M.J., Chan, F., Lanctot, K., Herrmann, N., McCullagh, S., \& Feinstein, A. (2008). An open-label study of citalopram for major depression following traumatic brain injury. Journal of Psychopharmacology, 22(8), 860-864.

Rapoport, M.J., Kiss, A., \& Feinstein, A. (2006). The impact of major depression on outcome following mildto-moderate traumatic brain injury in older adults. Journal of Affective Disorders, 92, 273-276.

Rapoport, M.J., McCullagh, S., Streiner, D., \& Feinstein, A. (2003a). Age and major depression after mild traumatic brain injury. American Journal of Geriatric Psychiatry, 11(3), 365-369.

Rapoport, M.J., McCullagh, S., Streiner, D., \& Feinstein, A. (2003b). The clinical significance of major depression following mild traumatic brain injury. Psychosomatics, 44(1), 31-37.

Rapoport, M.J., Mitchell, R.A., McCullagh, S., Herrmann, N., Chan, F., Kiss, A., ... Lanctôt, K.L. (2010). A randomized controlled trial of antidepressant continuation for major depression following traumatic brain injury. Journal of Clinical Psychiatry, 71(9), 1125-1130.

Robins, L.N., Helzer, J.E., Croughan, J., \& Ratcliff, K.S. (1981). National institute of mental health diagnostic interview schedule. its history, characteristics, and validity. Archives of General Psychiatry, 38(4), 381389.

Saran, A.S. (1985). Depression after minor closed head injury: Role of dexamethasone suppression test and antidepressants. Journal of Clinical Psychiatry, 46(8), 335-338.

Schoenberger, N.E., Shif, S.C., Esty, M.L., Ochs, L., \& Matheis, R.J. (2001). Flexyx neurotherapy system in the treatment of traumatic brain injury: An initial evaluation. Journal of Head Trauma Rehabilitation, 16(3), 260-274.

Seel, R.T., \& Kreutzer, J.S. (2003). Depression assessment after traumatic brain injury: An empirically based classification method. Archives of Physical Medicine and Rehabilitation, 84(11), 1621-1628.

Seel, R.T., Macciocchi, S., \& Kreutzer, J.S. (2010). Clinical considerations for the diagnosis of major depression after moderate to severe TBI. Journal of Head Trauma Rehabilitation, 25(2), 99-112.

Sheehan, D.V., Lecrubier, Y., Sheehan, K.H., Amorim, P., Janavs, J., Weiller, E., ... Dunbar, G.C. (1998). The mini-international neuropsychiatric interview (M.I.N.I.): The development and validation of a structured diagnostic psychiatric interview for DSMIV and ICD-10. Journal of Clinical Psychiatry, 59(Suppl. 20), 22-33; quiz 34-57.

Simpson, G.K., Tate, R.L., Whiting, D.L., \& Cotter, R.E. (2011). Suicide prevention after traumatic brain in- jury: A randomized controlled trial of a program for the psychological treatment of hopelessness. Journal of Head Trauma Rehabilitation, 26(4), 290-300.

Svendsen, H.A., Teasdale, T.W., \& Pinner, M. (2004). Subjective experience in patients with brain injury and their close relatives before and after a rehabilitation programme. Neuropsychological Rehabilitation, 14(5), 495-515.

Taylor, D., Meader, N., Bird, V., Pilling, S., Creed, F., Goldberg, D., ... Pharmacology Subgroup of the National Institute for Health and Clinical Excellence Guideline Development Group for Depression in Chronic Physical Health Problems (2011). Pharmacological interventions for people with depression and chronic physical health problems: Systematic review and meta-analyses of safety and efficacy. British Journal of Psychiatry: The Journal of Mental Science, 198(3), 179-188.

Tiersky, L.A., Anselmi, V., Johnston, M.V., Kurtyka, J., Roosen, E., Schwartz, T., ... Deluca, J. (2005). A trial of neuropsychologic rehabilitation in mildspectrum traumatic brain injury. Archives of Physical Medicine and Rehabilitation, 86(8), 1565-1574.

Topolovec-Vranic, J., Cullen, N., Michalak, A., Ouchterlony, D., Bhalerao, S., Masanic, C., ... Cusimano, M.D. (2010). Evaluation of an online cognitive behavioural therapy program by patients with traumatic brain injury and depression. Brain Injury, 24(5), $762-772$.

Turner-Stokes, L., Hassan, N., Pierce, K., \& Clegg, F. (2002). Managing depression in brain injury rehabilitation: The use of an integrated care pathway and preliminary report of response to sertraline. Clinical Rehabilitation, 16(3), 261-268.

Vanderploeg, R.D., Curtiss, G., Luis, C.A., \& Salazar, A.M. (2007). Long-term morbidities following selfreported mild traumatic brain injury. Journal of Clinical and Experimental Neuropsychology, 29(6), 585598.

Whelan-Goodinson, R., Ponsford, J., Johnston, L., \& Grant, F. (2009). Psychiatric disorders following traumatic brain injury: Their nature and frequency. Journal of Head Trauma Rehabilitation, 24(5), 324 332.

Wing, J.K., Babor, T., Brugha, T., Burke, J., Cooper, J.E., Giel, R., ... Sartorius, N. (1990). SCAN. schedules for clinical assessment in neuropsychiatry. Archives of General Psychiatry, 47(6), 589-593.

Wroblewski, B.A., Joseph, A.B., \& Cornblatt, R.R. (1996). Antidepressant pharmacotherapy and the treatment of depression in patients with severe traumatic brain injury: A controlled, prospective study. Journal of Clinical Psychiatry, 57(12), 582587.

Zigmond, A.S., \& Snaith, R.P. (1983). The hospital anxiety and depression scale. Acta Psychiatrica Scandinavica, 67(6), 361-370. 